\title{
The Incidence Rate and Economic Burden of Catheter-related Bloodstream Infection (CRBSI): a Single center, Retrospective Study
}

\section{Yibo Zhang}

Ruijin Hospital, Shanghai Jiao Tong University School of Medicine

\section{Yichen Wang}

Ruijin Hospital, Shanghai Jiao Tong University School of Medicine

\section{Dake Shi}

Ruijin Hospital, Shanghai Jiao Tong University School of Medicine

\section{Qun Wang}

Ruijin Hospital, Shanghai Jiao Tong University School of Medicine

\section{Shirui $\mathrm{Xu}$}

Shanghai Fenglin Clinical Laboratory Co. Itd

\section{Zhujun Cao}

Ruijin Hospital, Shanghai Jiao Tong University School of Medicine

\section{Yaping Ai}

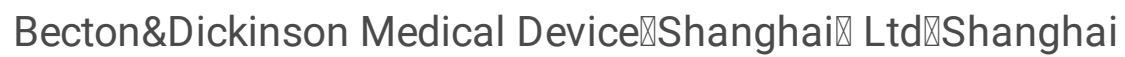

\section{Erzhen Chen}

Ruijin Hospital, Shanghai Jiao Tong University School of Medicine

\section{Yuxing Ni}

Ruijin Hospital, Shanghai Jiao Tong University School of Medicine

Yumin XU ( $\nabla$ xym121@163.com)

Ruijin Hospital, Shanghai Jiao Tong University School of Medicine

\section{Research Article}

Keywords: CRBSI, LOS, Cost, Antibiotics, ICU

Posted Date: June 14th, 2022

DOI: https://doi.org/10.21203/rs.3.rs-1334417/v2

License: (c) (i) This work is licensed under a Creative Commons Attribution 4.0 International License.

Read Full License 


\section{Abstract}

\section{Background}

Indwelling central venous catheters (CVCs) have the potential to lead to catheter related blood stream infection (CRBSI). We report the findings of a single center, retrospective matched case-control study that assessed the incidence rate of CRBSI in ICUs and attributable length of stay (LOS), costs among patients with CRBSI in ICU in Shanghai, China.

\section{Methods}

The study was carried out in several ICUs, with a total of 2300 beds of 3 tertiary-level hospital affiliated to Shanghai Jiao Tong University. The Department of Infection Control performs routinely surveillance for CRBSI on ten different ICUs.

\section{Results}

The CRBSI incidence rate of hematology ICU, which was the highest, was 4.43 per 1000 CVC-days. 51 patients were successfully matched with control patients. The average cost in the CRBSI group was $\$$ 67,923 which was significantly higher $(P<0.001)$ than that in the control group. The total average cost attributable to CRBSI was $\$ 33,227$.

Conclusions

The cost of antibiotics was closely related to the incidence of CRBSI.

\section{Introduction}

Central venous catheters (CVC) are commonly used in critically ill patients in Intensive Care Unit (ICU) and offer several advantages for treatment. However, indwelling CVCs have the potential to lead to several complications such as catheter related blood stream infection (CRBSI), phlebitis, catheter blockage, catheter exfoliation and venous thrombosis(1). Several studies have demonstrated that CRBSI is related with increased patient morbidity, prolonged length of stay and increased costs(2-5). Decreasing the rate of CRBSI is also one of the 2021 Chinese national medical quality and safety improvement goals(6). Therefore, it is important to understand the current situation as well as the disease burden of CRBSI. Due to limited research in this area, our goal of this study is to fill this gap. In this study, we report the findings of a single center, retrospective matched case-control study that assessed the incidence rate of CRBSI in ICUs and attributable length of stay (LOS), costs among patients with CRBSI in ICU in Shanghai, China.

\section{Methods}

Setting. The study was carried out in several ICUs, with a total of 2300 beds of 3 tertiary-level hospital affiliated to Shanghai Jiao Tong University Medical School. The infection control department performs 
routinely surveillance for CRBSI on ten different ICUs.

\section{Definition}

The US Center for Disease Control (CDC) defines CRBSI as a pathogen isolated from a blood culture (a single culture for organisms which are not commonly present on skin and two or more blood culture for organisms which are commonly present on the skin) in a patient with signs and symptoms of infection, who has a central line in place at the time of the identification of the infection or within $48 \mathrm{~h}$ before the infection develops. Such infections cannot be related to any other source for infection that the patient may have or was incubating when the patient was admitted(7-9).

Study design. The study is separated into two parts. In the first part, we studied the incidence rate of CRBSI in ten ICUs and the demographic characteristics related with patients with CRBSI.

Using a matched case-control design, we study the cost attributable to CRBSI compared with patients without CRBSI in our surveilled ICUs in the second part.

All patients with CVC in ten ICUs from July 2013 to June 2018 were studied. In the following, patients with CRBSI are classified as cases and patients without CRBSI are classified as potential controls. Cases and controls were found by continuous surveillance by our infection control members with many years of experience.

Information for each patient is gathered from Hospital Infection Database. For all patients included in our study, the following demographic characteristics were collected: age, gender, date of ICU admission, length of stay (LOS), critical care score(CCS) and the ICU types. ICU types is categorized as Cardiology ICU(CICU), Surgical ICU(SICU), General ICU(GICU), VIP ICU(VICU), Respiratory ICU(RICU), Hematology ICU $(\mathrm{HICU})$.

Controls and matching criteria. Cases were defined as patients who were 18 years or older at the time of hospital admission and who acquired a CRBSI on one of the analyzed ICUs. They had to be admitted to the hospital from July 2015 to June 2018.

All patients with CVC who were admitted to ICUs from July 2013 to June 2018 were considered potential controls. Control patients who were clinically diagnosed with BSI or who could be diagnosed with BSI following the $C D C$ definitions were excluded from the study.

A matched (1:1) case-control study design was utilized. A total of five key variables were used in the matching procedure, including gender, ICU, age ( \pm 5 year.), CCS $( \pm 2 \mathrm{pt})$, and date of ICU admission $( \pm$ 1 year).

Assessment of cost attributable to CRBSI. The cost data were derived from the actual hospital expenditure and were provided by the financial control department of the hospital. The duration from the time of patient admission to the day of discharge was selected. The costs of patients with CRBSI were 
analyzed and compared with those of the patients without CRBSI. The difference in cost between the two groups during the hospitalization was calculated as the cost attributable to CRBSI. This difference included the total cost and eight specific cost categories: 1) antimicrobial agent, 2) Non-antimicrobial agent ,3) bed, 4) health care technical service, 5) nursing, 6) laboratory testing, 7) medical material, 8) others.

The analysis of the drug included only western medicine. Bed cost was defined as the payment of rent for bed during hospitalization. Health care technical service included physical examination, medical disposition, and group consultation. Nursing cost means cost of nursing service. Laboratory testing cost included a routine blood test, and a bacterial culture. Medical material was defined as the medical consumables and equipment used in the hospital such as catheters and sutures. Others involved other expenses for diagnosis and treatment.

Statistical analysis. The incidence of CRBSI is defined as: CRBSI cases/ patients with CVC* 100 .The incidence rate of CRBSI is defined as: CRBSI cases/ CVC-days ${ }^{\star} 1,000$. Each day, a patient has one or more central line counts as one catheter day. When calculating the rate, it is expressed as the number of CRBSI per 1000 CVC days.

The differences in age and critical care score in the case group and all CVC-patients group were evaluated by Student's t-test. The differences in gender and incidence of CRBSI were evaluated by Chi-square test.

The matching method is nearest neighbor matching with replacement. If more than one control cases met the matching criteria, the order of selection criteria was critical care score, age and date of ICU admission.

The difference in age in the case and control group after the matching procedure was evaluated by Student's t-test. The differences in cost and hospital day for the patients between the two groups were tested by the Wilcoxon signed-rank test.

\section{Results}

Evaluation of the incidence and incidence rate. There are 8,019 patients with CVC in the ICUs from July 2013 to June 2018, of which a total of 82 patients with CRBSI were involved in the case group from 2015 to 2018 . The CVC days were $64,477.8$ CVC-days. The demographic characteristics and patient distribution is shown in Table 1. Nearly 1/4 patients with CRBSI were in Cardiac ICU. which involved more than half of patients with CVC. The total incidence and incidence rate of CRBSI was $1.08 \%$ and 1.27 per CVC days. The incidence rate of hematology ICU was highest, which was 4.43 per 1000 CVC-days. Other data is shown in Table 2. The pathogen of CRBSI is shown in Table 3. 
Table 1

Demographic characteristics and distribution of patients

\begin{tabular}{|c|c|c|}
\hline & Patients with CVC $(n=8,019)$ & No. CRBSI $(n=82)$ \\
\hline \multicolumn{3}{|c|}{ Demographic characteristics } \\
\hline Age & $59.80( \pm 16.9)$ & $53.30( \pm 18.1)$ \\
\hline Female & $2,902(36.2 \%)$ & $22(26.8 \%)$ \\
\hline $\operatorname{ccs}$ & $12.47( \pm 5.5)$ & $2.54( \pm 4.4)$ \\
\hline \multicolumn{3}{|l|}{ ICU Departments } \\
\hline Cardiac ICU & $4362(54.40 \%)$ & $20(24.39 \%)$ \\
\hline Respiratory ICU & $149(1.86 \%)$ & $1(1.22 \%)$ \\
\hline Surgical ICU & $1868(23.29 \%)$ & $16(19.51 \%)$ \\
\hline General ICU & $869(10.84 \%)$ & $11(13.41 \%)$ \\
\hline Hematology ICU & $498(6.21 \%)$ & $33(40.24 \%)$ \\
\hline VIP ICU & $273(3.40 \%)$ & $1(1.22 \%)$ \\
\hline
\end{tabular}

Table 2

Incidence rate of CRBSI in ten ICUs

\begin{tabular}{|llllll|}
\hline & $\begin{array}{l}\text { No. } \\
\text { CRBSI }\end{array}$ & $\begin{array}{l}\text { Patients with } \\
\text { CVC }\end{array}$ & $\begin{array}{l}\text { CVC- } \\
\text { days }\end{array}$ & $\begin{array}{l}\text { Incidence rate(per 1000 CVC- } \\
\text { days) }\end{array}$ & $\begin{array}{l}\text { Incidence } \\
\text { (\%) }\end{array}$ \\
\hline Cardiac ICU & 20 & 4362 & 14785.8 & 1.35 & $0.46 \%$ \\
\hline $\begin{array}{l}\text { Respiratory } \\
\text { ICU }\end{array}$ & 1 & 149 & 1986.3 & 0.50 & $0.67 \%$ \\
\hline Surgical ICU & 16 & 1868 & 19190.5 & 0.83 & $0.86 \%$ \\
\hline General ICU & 11 & 869 & 11798.2 & 0.93 & $1.27 \%$ \\
\hline $\begin{array}{l}\text { Hematology } \\
\text { ICU }\end{array}$ & 33 & 498 & $9,383.8$ & 3.52 & $6.63 \%$ \\
\hline VIP ICU & 1 & 273 & 7333.2 & 0.14 & $0.37 \%$ \\
\hline Overall & 82 & 8019 & 64477.8 & 1.27 & $1.02 \%$ \\
\hline
\end{tabular}


Table 3

Pathogens of CRBSI

\begin{tabular}{|llllll|}
\hline Pathogen & $\begin{array}{l}\text { No. } \\
\text { CRBSI }\end{array}$ & $\begin{array}{l}\text { Patients with } \\
\text { CVC }\end{array}$ & $\begin{array}{l}\text { CVC- } \\
\text { days }\end{array}$ & $\begin{array}{l}\text { Incidence rate(per 1000 CVC- } \\
\text { days) }\end{array}$ & $\begin{array}{l}\text { Incidence } \\
\text { (\%) }\end{array}$ \\
\hline Overall & 82 & 8,019 & $64,477.8$ & 1.27 & $1.02 \%$ \\
\hline Bacteria & 70 & 949 & $17,387.7$ & 4.03 & $7.38 \%$ \\
\hline fungus & 23 & 239 & $4,817.0$ & 4.77 & $9.62 \%$ \\
\hline
\end{tabular}

Fundamental results of matching. According to the matching criteria, 51 patients were successfully matched with control patients (the information of the other 31 patients was incomplete or could not be matched properly). The average age of the case group was $50.0 \pm 19.0$ years old, and that of the control group was $50.3 \pm 18.7$ years old $(P=0.18)$. The average critical score of the case group was $12.6 \pm 4.6$ points, and that of the control group was $12.4 \pm 4.6$ points $(P=0.07)$. Eight ICUs were involved, including cardiac ICU (11 pairs), general ICU (7 pairs), surgical ICU (7 pairs), Hematology ICU (25 pairs). The demographic characteristics and department distribution of patients of matched group are shown in Table 4.

Table 4

Demographic characteristics and department distribution of patients of matched group

\begin{tabular}{|lll|}
\hline \multicolumn{2}{|c|}{ Patients with CVC $(\mathbf{n}=\mathbf{5 1})$} & No. CRBSI $(\mathbf{n}=\mathbf{5 1})$ \\
\hline Demographic characteristics & & \\
\hline Age & $50.0( \pm 19.0)$ & $50.3( \pm 18.7)$ \\
\hline Female & $17(33.3 \%)$ & $17(33.3 \%)$ \\
\hline ICU Departments & $12.6( \pm 4.6)$ & $12.4( \pm 4.6)$ \\
\hline Cardiac ICU & & \\
\hline Hematology ICU & $11(21.6 \%)$ & $11(21.6 \%)$ \\
\hline General ICU & $25(49.1 \%)$ & $25(49.1 \%)$ \\
\hline Surgical ICU & $7(13.7 \%)$ & $7(13.7 \%)$ \\
\hline Respiratory ICU & $7(13.7 \%)$ & $7(13.8 \%)$ \\
\hline
\end{tabular}

Evaluation of the hospital day. The median hospital days in the CRBSI group were 56.0 days, which were significantly higher $(P<0.001)$ than that in the control group (36.0 days). The median additional hospital days were 20.0 days. 
Evaluation of the cost attributable to CRBSI. The average cost in the CRBSI group was $\$ 67,923$ which was significantly higher $(P<0.001)$ than that in the control group. The total average cost attributable to CRBSI was $\$ 33,696$. Furthermore, the differences between the antimicrobial agent, other drug, bed, health care technical service, nursing, laboratory testing, medical material, others of the two groups were $\$ 9,039$, $\$ 9,742, \$ 1,539, \$ 21, \$ 365, \$ 5,330, \$ 4,230$ and $\$ 3,430$, respectively. (Table 5)

Table 5

Medical costs attributable to CRBSI (\$)

\begin{tabular}{|c|c|c|c|c|}
\hline & $\begin{array}{l}\text { Average cost in the } \\
\text { CRBSI group }\end{array}$ & $\begin{array}{l}\text { Average cost in the } \\
\text { control group }\end{array}$ & $\begin{array}{l}\text { Medical cost } \\
\text { attributable to CRBSI }\end{array}$ & $\begin{array}{l}\mathrm{P} \\
\text { Value }\end{array}$ \\
\hline Drug & 38,288 & 19,507 & 18,781 & $\dot{0} 01$ \\
\hline $\begin{array}{l}\text { Antimicrobial } \\
\text { agent }\end{array}$ & 21,073 & 12,033 & 9,030 & $\hat{0}_{0.01}$ \\
\hline $\begin{array}{l}\text { Non-antimicrobial } \\
\text { agent }\end{array}$ & 17,215 & 7,473 & 9,742 & $\dot{0} 01$ \\
\hline $\begin{array}{l}\text { Total medical } \\
\text { cost }\end{array}$ & 29,636 & 14,720 & 14,915 & $\begin{array}{l}< \\
0.001\end{array}$ \\
\hline Bed & 4,608 & 3,069 & 1,539 & $\dot{0.05}$ \\
\hline $\begin{array}{l}\text { health care } \\
\text { technical service }\end{array}$ & 135 & 115 & 21 & $\overrightarrow{0} .05$ \\
\hline nursing & 783 & 417 & 365 & $\dot{0.001}$ \\
\hline laboratory testing & 10,630 & 5,529 & 5,330 & $\dot{0.001}$ \\
\hline medical material & 7,450 & 3,220 & 4,230 & $\dot{0} 001$ \\
\hline others & 6,030 & 2,601 & 3,430 & $\dot{0.001}$ \\
\hline Total & 67,923 & 34,227 & 33,696 & $\begin{array}{l}< \\
0.001\end{array}$ \\
\hline
\end{tabular}

\section{Discussion}

Central vascular access is quite vital for ICU patients and about $90 \%$ of bloodstream infection is related to $\mathrm{CVC}(10)$. The overall incidence and incidence rate of CRBSI of this study was $1.08 \%$ and 1.27 per 1000 CVC-days, which is similar to the CRBSI rate of 1.1 per 1000 CVC-days in the United States as determined by the CDC NSHN and lower than the rate of 3.7 in Europe reported by the European Centre for Disease Prevention and Control, compared with developed countries(11). Chopdekar K., et al reported the average 
CRBSI incidence at 9.26 per 1000 CVC-days in ICUs in a tertiary care teaching hospital in Mumbai(12). However, the rate of Hematology ICU was approximately 3 -fold higher than the overall rate. All patients in Hematology ICU had a bone marrow transplantation, who were in presence of inhibition of body immunity. They were extremely susceptible that any bacteria in the environment. The incidence of CRBSI varies in different areas, which might be related to the patient's condition, catheter selection, catheter placement sites and catheter maintenance, or the definition of diagnostic criteria for CRBSI might varies.

ICU patients are usually in complex and critical condition. Due to the needs of monitoring and treatment, a variety of invasive operations such as central venous catheters are usually required. All these factors will increase the possibility of blood stream infection in patients. Once bloodstream infection occurs, especially CRBSI, the patient's condition rapidly becomes worse, causing sepsis easily and even leading to death, which brings great challenges to medical staffs. Studies have shown that the mortality rate of ICU patients with CRBSI is higher than that of patients without CRBSI(13).

This study found 82 cases of CRBSI occurred in 6 ICUs from July 2013 to June 2018 in total, among which the incidence of CRBSI is highest in Hematology ICU (6.63\% and 3.52 per 1000 catheter days, respectively), which is higher than Rabensteiner's study(2.6 per 1000 catheter days)(14). The lowest is in VIP ICU (0.37\% and 0.14 per 1000 catheter days, respectively). Patients with malignant hematologic diseases are mainly treated in the hematology ICU. They are in immunosuppressed state, and broadspectrum antibiotics are routinely used to prevent infection. Since all patients need chemotherapy treatment, almost all of them have central venous catheters during hospitalization, which increases the risk of infection, leading to the incidence of CRBSI higher than these of other ICUs. It should be noted that although the incidence of CRBSI in hematology ICU in this study was higher than these in other ICUs, however, it was far lower than that in the study of Jing Xueming(15) and Chen Xing(16), which may be related to catheter site selection.

Mostly elderly patients are admitted in VIP ICU. Although their general conditions are not good, the high ratio of the ICU medical staff, adequate equipment security, and in-depth understanding of patients' situation can bring individualized diagnosis and treatment to these patients. Those advantages can ensure every patient's condition stable, thus the incidence of CRBSI in VIP ICU is the lowest.

In this study, the incidence of CRBSI in cardiac ICU was the second highest, while the incidence of CRBSI per 1000 catheter days was the second lowest. The reason might be that most patients were observed in cardiac ICU for 48 hours and often transferred between cardiothoracic ICU and general wards. The high beds turnover rate leads to very low average number of days of catheterization. Thus, there is a mismatch between the incidence of CRBSI and the infection rate per 1000 catheter days.

We found the median hospital day attributable to CRBSI was 20.0 days, which was much higher than the extra length of stay (7 days) of Higuera's and Leistner 's study $(17,18)$. Rosenthal's study in Argentina in six ICUs among three hospitals found that the hospital day attributable to CRBSI was 11.9 days(19). Dimick found severe surgical ICU patients, the attributable hospital days to CRBSI was 20 days, which was quite similar as this study(20). 
Studies at home and abroad have shown that CRBSI can prolong the length of stay in ICU and the total length of stay. However, the results of different studies vary greatly due to the differences in medical charge patterns and statistical methods such as patient grouping in different regions. If patients are simply divided into infected and uninfected groups, and then the difference in average length of stay between the two groups is compared, it will produce biased estimates due to the influence of patients' basic diseases and physical states. By means of matching, confounding factors are controlled between the case group and the control group, which can relatively reduce the differences between the underlying diseases and other factors of the patients

Multiple studies have shown that the extension of hospital stay is one of the important factors affecting the increase of nosocomial infection $(21,22)$. In this study, patients with CRBSI extended the hospital stay by an average of 20 days compared with patients without CRBSI. Therefore, the hospital stay of patients is positively correlated with the occurrence of nosocomial infection, and the two may be mutually causal. Patients with CRBSI need targeted treatment, increased use of antimicrobial agents and corresponding supportive treatment, so as to prolong the course of disease, thus leading to reduced ICU bed turnover rate and increased bed utilization rate. The excessively high utilization rate of hospital beds, on the one hand, leads to the delay of treatment of patients in need of treatment because there is no idle bed, and increases the potential of doctor-patient disputes(23-26).

This study is the second to report the estimated additional costs of CRBSI of ICU in China. The additional average medical costs of each case of CRBSI in the ICU were $\$ 67,923$. The medical average cost attributable to CRBSI is $\$ 18,781$, for which antibiotic agents mostly account, almost twice as much as the cost in the control group.

Dimick's study in US found the additional cost of CRBSI in surgical ICU was $\$ 11,523$ to $\$ 165,735(20)$. David et al. found the hospital costs attributable to CRBSI in ICU in Canada was $\$ 11,971(27)$. Another study on the cost of CRBSI in Canada found the cost of CRBSI acquired ICU is $\$ 12,321(28)$.

Since October 1st 2008, the United States has stipulated that the medical costs caused by CRBSI are paid by the hospital, and patients do not have to pay. Currently China has not established similar regulations. In China, most of the special antibiotics for the antimicrobial resistant bacteria infection treatment, such as carbapenem-resistant Enterobacteriaceae bacteria, are quite expensive and not covered by the basic medical reimbursement.

First of all, the occurrence of CRBSI will increase the cost of microorganism culture and drug sensitivity test of patients. In addition, the cost of antibiotics such as lineczolid, tigecycline and polymyxin used in the early empirical treatment and targeted treatment of pathogenic bacteria is quite expensive, which greatly increases the cost of treatment for patients. The overuse of antibiotics not only consumes excessive health resources, but also increases the risk of nosocomial infection and the emergence and spread of drug-resistant bacteria. Therefore, reducing the incidence of CRBSI can not only reduce the disease burden of patients, but also reduce the occurrence of nosocomial infection and the spread of 
drug-resistant bacteria, which is of great significance for improving the quality of medical care and ensuring the safety of patients.

Once the patient of ICU got a CRBSI, lots of high-level antibiotic agents would be used, which were substantially expensive and less covered by medical insurance service. Our study innovatively considered the cost of antibiotics which was closely related to the incidence of CRBSI. As a proactive anticipative strategy, microscopic examination of CVC blood samples might be used to anticipate CRBSI in an earlier stage(29).

Our study has some limitations. 1)All information of patients with CVC was recorded lack of intubation position. The rates of different central lines were unavailable. 2) We cannot analyze the drug used for different organism, cause several antibiotic agents could be used for treatment of certain organism, and the date of prescription was not recorded. 3) Other infections were not excluded, which need to be considered in the future research.

\section{Conclusions}

In conclusion, the cost of antibiotics was closely related to the incidence of CRBSI. Our study suggests that measures should be taken to prevent the occurrence of CRBSI, not only to reduce the cost of CRBSI and antibiotics, but also to control the consequences of antibiotic abuse.

\section{Abbreviations}

CVC: central venous catheters; CRBSI: Catheter-related Bloodstream Infection; ICU: Intensive Care Unit.

\section{Declarations}

\section{Ethics approval and consent to participate}

Approved by the Ethics Committee of Ruijin Hospital, Jiaotong University School of Medicine.

\section{Consent for publication}

Not applicable.

Availability of data and materials

Both Yibo Zhang and Yichen Wang have full access to the data used for this manuscript. The data that support the findings of this study are available from the corresponding author, upon reasonable request.

\section{Competing interests}

The authors declare that they have no competing interests.

\section{Funding}

Page 10/13 
This work was funded by the National Natural Science Foundation of China (81670569), Shanghai Shen Kang Hospital Development Center (SHDC2020CR6025), Shanghai Jiaotong University School of Medicine Hospital Infection Committee 2022.

\section{Authors' contributions}

All authors contributed to the study design and conception. Data collection was performed by Yibo Zhang. Data analysis was performed by Yichen Wang. The expert panel consisted of Yumin XU, Yuxing $\mathrm{Ni}$, Erzhen Chen. The first draft of the manuscript was written by Yibo Zhang and Yichen Wang. The manuscript was reviewed by Dake Shi and Qun Wang. The study was coordinated by Shirui Xu, Zhujun Cao and Yaping Ai. All authors commented on earlier versions of the manuscript approved the final manuscript.

\section{Acknowledgements}

Not applicable

\section{References}

1. van der Kooi T, Sax H, Pittet D, van Dissel J, van Benthem B, Walder B, et al. Prevention of hospital infections by intervention and training (PROHIBIT): results of a pan-European cluster-randomized multicentre study to reduce central venous catheter-related bloodstream infections. Intensive care medicine. 2018;44(1):48-60.

2. Brown R, Burke D. The hidden cost of catheter related blood stream infections in patients on parenteral nutrition. Clinical nutrition ESPEN. 2020;36:146-9.

3. Saliba P, Hornero A, Cuervo G, Grau I, Jimenez E, García D, et al. Mortality risk factors among non-ICU patients with nosocomial vascular catheter-related bloodstream infections: a prospective cohort study. Journal of Hospital Infection. 2018;99(1):48-54.

4. Burden S, Hemstock M, Taylor M, Teubner A, Roskell N, MacCulloch A, et al. The impact of home parenteral nutrition on the burden of disease including morbidity, mortality and rate of hospitalisations. Clinical nutrition ESPEN. 2018;28:222-7.

5. Foda MA, Khalil GM, Gaddoue IM, Azqul MM. Relation between Health Care Providers Compliance with Central Venous Catheter Care Bundle Elements and The Length of Stay in Cardiac Care Units, National Heart Institute. Egyptian Journal of Community Medicine. 2018;36(4).

6. National Health Commission of PRC. 2021 National Medical Quality and Safety Improvement Goals. http://www.gov.cn/zhengce/zhengceku/2021-02/22/content_5588240.htm. Updated February 9, 2021.

7. Gastmeier P, Geffers C, Brandt C, Zuschneid I, Sohr D, Schwab F, et al. Effectiveness of a nationwide nosocomial infection surveillance system for reducing nosocomial infections. The Journal of hospital infection. 2006;64(1):16-22. 
8. Dudeck MA, Weiner LM, Allen-Bridson K, Malpiedi PJ, Peterson KD, Pollock DA, et al. National Healthcare Safety Network (NHSN) report, data summary for 2012, Device-associated module. Am J Infect Control. 2013;41(12):1148-66.

9. Chopdekar K, Chande C, Chavan S, Veer P, Wabale V, Vishwakarma K, et al. Central venous catheterrelated blood stream infection rate in critical care units in a tertiary care, teaching hospital in Mumbai. Indian journal of medical microbiology. 2011;29(2):169-71.

10. Yin D-R, Su H-P, Nian Q-P. Epidemiological characteristics and risk factors of catheter-related bloodstream infections in ICU patients. Chinese Journal of Nosocomiology. 2017;27(019):4375-7.

11. Rabensteiner J, Theiler G, Duettmann W, Zollner-Schwetz I, Hoenigl M, Valentin T, et al. Detection of central venous catheter-related bloodstream infections in haematooncological patients. European journal of clinical investigation. 2015;45(8):824-32.

12. Xue-ming Jing, Ling Li1, Xiao-ping Mei, et al. Clinical characteristics and risk factors of catheterrelated bloodstream infections in patients with hematologic malignancies. China Journal of Modern Medicine. 2016;26(01):119 - 23.

13. Chen Xing, Ji M, Liu J et al.. Analyse the influence and risk factors of venipuncture mode of catheterrelated bloodstream infections in patients with hematologic malignancies. Journal of North Sichuan Medical College. 2018;v.33;No.161(02):133-7.

14. Higuera F, Rangel-Frausto MS, Rosenthal VD, Soto JM, Castañon J, Franco G, et al. Attributable cost and length of stay for patients with central venous catheter-associated bloodstream infection in Mexico City intensive care units: a prospective, matched analysis. Infection control and hospital epidemiology. 2007;28(1):31-5.

15. Leistner R, Hir semann E, Bloch A, Gastmeier P, Geffers C. Costs and prolonged length of stay of central venous catheter-associated bloodstream infections (CVC BSI): a matched prospective cohort study. Infection. 2014;42(1):31-6.

16. Rosenthal VD, Guzman S, Migone O, Crnich CJ. The attributable cost, length of hospital stay, and mortality of central line-associated bloodstream infection in intensive care departments in Argentina: A prospective, matched analysis. Am J Infect Control. 2003;31(8):475-80.

17. Dimick JB, Pelz RK, Consunji R, Swoboda SM, Hendrix CW, Lipsett PA. Increased resource use associated with catheter-related bloodstream infection in the surgical intensive care unit. Archives of surgery (Chicago, III: 1960). 2001;136(2):229 - 34.

18. Stewart S, Robertson C, Pan J, et al. Impact of healthcare-associated infection on length of stay[J]. Journal of Hospital Infection, 2021, 114:23-31.

19. Wang L, Zhou K-H, Chen W, Yu Y, Feng S-F. Epidemiology and risk factors for nosocomial infection in the respiratory intensive care unit of a teaching hospital in China: A prospective surveillance during 2013 and 2015. BMC infectious diseases. 2019;19(1):1-9.

20. Liu X, Wang D-F, ChenQ-M. Comparison of critical care resources between second-class hospitals and third-class hospitals in Guizhou Province of China. Chinese Critical Care Medicine. 2020;032(002):230-4. 
21. Li W, lu X, Cao J, et al. Human Resources Allocation for Nursing in Intensive Care Unit. Hospital Administration Journal of Chinese Peoples Liberation Army. 2019;026(009):827-30.

22. He AJ, Qian J. Explaining medical disputes in Chinese public hospitals: the doctor-patient relationship and its implications for health policy reforms. Health Econ Policy Law. 2016 Oct;11(4):359-78.

23. Li D, Chen L. Work Stress,Work Family Conflict to Flexible Work Practice Attribution of ICU Nurses in Beijing. Chinese Journal of Social Medicine. 2021; 38(1):117-121.

24. Warren DK, Quadir WW, Hollenbeak CS, Elward AM, Cox MJ, Fraser VJ. Attributable cost of catheterassociated bloodstream infections among intensive care patients in a nonteaching hospital. Critical care medicine. 2006;34(8):2084-9.

25. Laupland KB, Lee H, Gregson DB, Manns BJ. Cost of intensive care unit-acquired bloodstream infections. The Journal of hospital infection. 2006;63(2):124-32.

26. Wagner J, Schilcher G, Zollner-Schwetz I, Hoenigl M, Valentin T, Ribitsch W, et al. Microbiological screening for earlier detection of central venous catheter-related bloodstream infections. European journal of clinical investigation. 2013;43(9):964-9.

27. Asad, Latif, Muhammad, Sohail, Halim, Peter, et al. Eliminating Infections in the ICU: CLABSI. Current Infectious Disease Reports. 2015;17(7):35.

28. Horan TC, Cic M, Dudeck MA. CDC/NHSN surveillance definition of health care-associated infection and criteria for specific types of infections in the acute care setting. American Journal of Infection Control. 2008;36(5):309-32.

29. None. Vital Signs: Central Line-Associated Blood Stream Infections-United States, 2001, 2008, and 2009. Annals of Emergency Medicine. 2011; 60(5):243-8. 